\title{
PERSPECTIVE
}

\section{Soil aggregates as massively concurrent evolutionary incubators}

\author{
Matthias C Rillig ${ }^{1,2}$, Ludo AH Muller ${ }^{1,2}$ and Anika Lehmann ${ }^{1,2}$ \\ ${ }^{1}$ Freie Universität Berlin, Institut für Biologie, Plant Ecology, Berlin, Germany and ${ }^{2}$ Berlin-Brandenburg \\ Institute of Advanced Biodiversity Research, Berlin, Germany
}

The ISME Journal (2017) 11, 1943-1948; doi:10.1038/ismej.2017.56; published online 14 April 2017

\section{Introduction}

Soil aggregation, a key component of soil structure, has mostly been examined from the perspective of soil management and the mediation of ecosystem processes such as soil carbon storage. However, soil aggregation is also a major factor to consider in terms of the fine-scale organization of the soil microbiome. For example, the physico-chemical conditions inside of aggregates usually differ from the conditions prevalent in the bulk soil and aggregates therefore increase the spatial heterogeneity of the soil. In addition, aggregates can provide a refuge for microbes against predation since their interior is not accessible to many predators. Soil aggregates are thus clearly important for microbial community ecology in soils (for example, Vos et al., 2013; Rillig et al., 2016) and for microbially driven biogeochemistry, and soil microbial ecologists are increasingly appreciating these aspects of soil aggregation. Soil aggregates have, however, so far been neglected when it comes to evolutionary considerations (Crawford et al., 2005) and we here propose that the process of soil aggregation should be considered as an important driver of evolution in the soil microbial community.

There are several features that make soil aggregates specifically interesting, and perhaps even unique, in terms of a setting for microbial evolution (Table 1). Soil aggregation is a continuous and dynamic process in which the formation and disintegration of individual micro- and macroaggregates are separated in time by periods of relative stability. Each individual soil aggregate may provide a unique environmental compartmentalization of the soil microbial community that is, to a large extent, isolated from its surroundings and that can be thought of as an

Correspondence: MC Rillig, Freie Universität Berlin, Institut für Biologie, Plant Ecology, Altenstein str. 6, D-14195 Berlin, Germany.

E-mail: matthias.rillig@fu-berlin.de

Received 30 November 2016; revised 5 February 2017; accepted

7 February 2017; published online 14 April 2017 'incubator' for microbial evolutionary change. Because of their isolation, different aggregates can be regarded as 'concurrent incubators' that allow enclosed microbial communities to pursue their own independent evolutionary trajectories during their lifetime ('incubation period'). The huge number of aggregates that exists at any moment in time validates their conceptualization as 'massively concurrent incubators' for microbial evolutionary change (Figure 1). Upon disintegration of soil aggregates ('incubation cycle ends'), formerly enclosed microbial communities are released and allowed to interact with the microbial community of the soil at large.

This combination of features (isolation, large number and relative stability) sets soil aggregates apart from other microbial habitats that may also provide temporary isolation of microbial communities, such as the animal intestinal tract and other parts of the animal body (see also Cordero and Datta, 2016), leaves, roots, and many aquatic habitats (Table 1). However, these habitats do not provide the same combination of extent of isolation, duration of isolation and number of concurrent 'incubators' as soil aggregates do.

We discuss these specific characteristics of soil aggregates next, before describing how evolutionary change in aggregates can occur and explaining how this system can be tackled empirically.

\section{Characteristics of soil aggregates important for microbial evolution}

Number of aggregates

Soil aggregates are exceptionally numerous. They are relatively small (microaggregates are $<250 \mu \mathrm{m}$, macroaggregates range from $250 \mu \mathrm{m}$ to a few $\mathrm{mm}$ in diameter) and soil essentially consists of packed aggregates and pore spaces. Thus, per square meter of soil surface in any terrestrial ecosystem, there is an enormous number of soil aggregates of various sizes. This is important, because each one of these aggregates can be viewed as an independent evolutionary 'incubator', having encased its own microbial community during its formation. 
Table 1 Qualitative comparison of various microbial habitat types in terms of features that are conducive to the formation of an evolutionary incubator for communities

\begin{tabular}{|c|c|c|c|c|}
\hline Microbial habitat type & $\begin{array}{l}\text { Absolute } \\
\text { number of } \\
\text { items in an } \\
\text { ecosystem } \\
\left(\text { per } \mathrm{m}^{2}\right)\end{array}$ & $\begin{array}{c}\text { Stability of } \\
\text { 'incubators' } \\
\text { per habitat } \\
\text { for periods of } \\
\text { months or } \\
\text { more }\end{array}$ & $\begin{array}{l}\text { Relatively } \\
\text { strong isola- } \\
\text { tion from } \\
\text { surrounding } \\
\quad \text { matrix }\end{array}$ & Eventual breakdown of enclosed 'incubator' \\
\hline Soil aggregate interiors & +++ & +++ & ++ & +++ (aggregate disintegration) \\
\hline $\begin{array}{l}\text { Aquatic environments (for example, ponds, } \\
\text { lakes, river, oceans) }\end{array}$ & 0 & +++ & 0 & 0 (often well mixed) \\
\hline $\begin{array}{l}\text { Aquatic environments-miniature } \\
\text { (for example, nectar droplets) }\end{array}$ & ++ & 0 & + & ++ (removal of droplet) \\
\hline $\begin{array}{l}\text { Animal host interiors (for example, } \\
\text { gastrointestinal) }\end{array}$ & + & + & + & $+++($ excretion $)$ \\
\hline $\begin{array}{l}\text { Animal host interiors (for example, other } \\
\text { animal tissues) }\end{array}$ & + & +++ & + & +++ (death) \\
\hline Host interiors (for example, plant organs) & + & ++ & 0 & $\begin{array}{c}++ \text { (shedding leaves, dying roots, } \\
\text { decomposition) }\end{array}$ \\
\hline $\begin{array}{l}\text { Object surfaces (for example, plant, animals, } \\
\text { built environment) }\end{array}$ & +++ & ++ & 0 & 0 (not applicable, since at surface) \\
\hline
\end{tabular}

\section{Relative isolation}

Soil aggregate interiors are quite isolated from the surrounding matrix. This is illustrated by very steep gradients in gas concentrations, for example, oxygen, from the inside to the outside of aggregates (Sexstone et al., 1985). This means that even the exchange of gases, let alone cells, is highly limited. Bacterial cells do possess mobility, and can move towards, and perhaps to a limited extent, also into aggregates (Dechesne et al., 2010), but the vast majority of the aggregate interior is blocked from dispersal. This degree of isolation is important for the trajectories of evolutionary changes that can occur inside of the aggregates (Vetsigian et al., 2011), which are largely unencumbered by migration.

\section{Stability}

Stability is important to allow time for evolutionary changes. By their very nature, aggregates are a structural component and thus a certain stability is inherent in their very existence. Aggregate stability is a property routinely measured in soil science. Soil science has also been concerned with describing and understanding the binding agents, and biotic players that mediate this stability. It is thus well known that these habitats can be stable at any given time, when examined at the level of entire aggregate populations. The dynamic nature of habitat turnover is less well understood, but the few measurements using tracers, most notably rare earth element oxides, have indicated that microaggregates are stable for months and macroaggregates for weeks to months (De Gryze et al., 2006). Most likely the range of aggregate stability times, depending on the site conditions, extends up to years.

\section{Breakdown}

The eventual breakdown of these evolutionary 'incubators' is also important, because this releases the entrapped microbial populations. Once aggregates are formed and stabilized by a variety of biota and chemical binding agents, they are subject to disintegration (Six et al., 2004). Disintegration can occur via a number of disintegrating forces, including water, tillage and others (for example, earthworms, pressure exerted by plant roots), and by soil organic matter decomposition. When an aggregate falls apart, the interior soil microbial community, with the evolutionary changes having occurred following the period of sequestration, is liberated and then this community is free to interact with the surrounding soil community (Rillig et al., 2016). This latter point is important, because now any evolutionary changes can become part of the 'mainstream' soil community, for example, via replacement of phylotypes or by horizontal gene transfer.

\section{Evolutionary change in soil aggregates}

We define microbial evolution in soil aggregates as any change in the relative allele frequencies or phylotype frequencies of the microbial populations enclosed within an aggregate; a change in allele frequency can arise by natural selection, migration, non-random mating (for example, outbreeding in heterothallic fungi) or genetic drift. In addition, de novo changes, for example, via horizontal gene transfer or by mutation, can occur within a lineage during the aggregate 'incubation' period. We divide our discussion of evolutionary forces into the three phases of the aggregate life cycle: aggregate formation, the stable aggregate period and the eventual disintegration (Figure 1a).

Aggregate formation: founder effects

During the formation of a soil aggregate, populations of microbial phylotypes present in the bulk soil are 

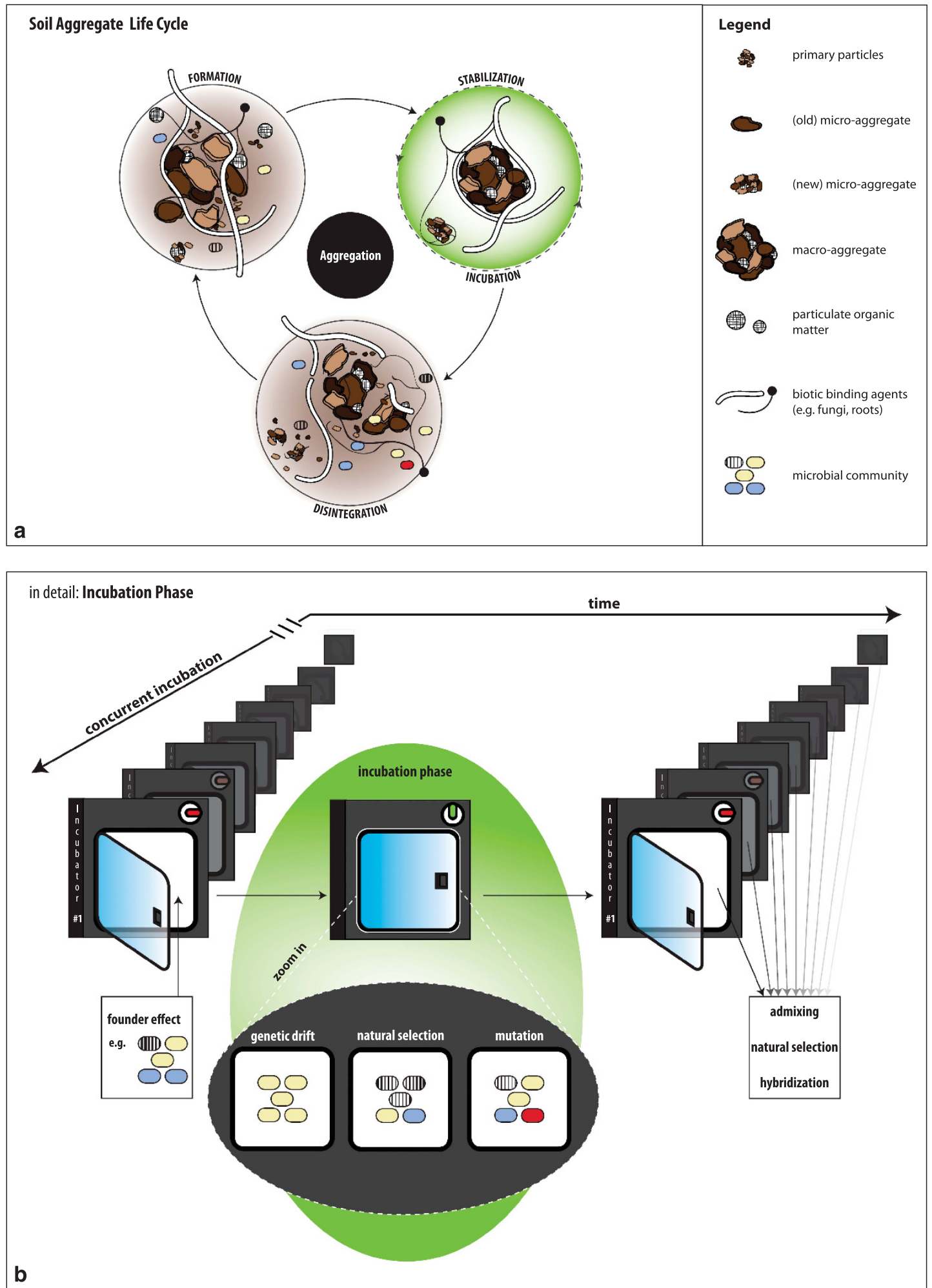

Figure 1 Evolutionary change in soil aggregates: the life cycle of a soil aggregate microbial 'incubator' (meaning a compartmentalized microbial habitat). (a) Depicts the dynamics of the soil aggregate life cycle, with the stage formation, stabilization/incubation and disintegration. (b) Depicts the evolutionary consequences of this soil aggregate life cycle, building on the metaphor of the 'incubator'. When the aggregate is formed ('incubator' is loaded), founder effects are of great importance. During the incubation phase (green shading in both $\mathbf{a}$ and $\mathbf{b}$ ), that is, while the aggregate is stable and its interior is isolated from the surroundings, a number of evolutionary processes take place, including genetic drift and natural selection, both of which will lead to a decrease in the genetic diversity in any single aggregate, and mutation, which may increase diversity. In the end, the previously aggregate-enclosed microbial populations are released into the soil at the time of aggregate breakdown ('incubator' opens), and then natural selection, admixture and hybridization can occur. Given that there are a myriad of concurrent 'incubation' events, the overall effect is likely an increase in soil microbial diversity. 
enclosed within the aggregate structure (Figure 1b). Although actual initial sizes of the enclosed microbial communities are unknown, a size range of several thousands to several millions of individuals is plausible depending on aggregate size and assuming a passive enclosure of microorganisms present in the bulk soil from which the aggregate was formed (Whitman et al., 1998). In any case, the enclosed population of any one phylotype is expected to be founded by a small number of individuals compared to the size of the source population in the bulk soil and founder effects are therefore likely. Such effects occur when only a fraction of the genetic diversity of the source population is represented within the newly founded population (genetic bottleneck) and the initial relative allele frequencies within the founded population are different from the frequencies in the source population. This may lead to low levels of genetic diversity within populations enclosed in new aggregates and high levels of genetic differentiation between populations enclosed in neighboring aggregates.

Inside the aggregate interior: genetic drift, natural selection and limited gene flow

Once formed, the aggregate constitutes an environment that is significantly different and isolated from the surrounding soil. Differences include altered availability of terminal electron acceptors, depletion of sources of organic carbon and nutrients, and potential accumulation of antibiotics or toxins (Vos et al., 2013). In addition, aggregate interiors are spatially heterogeneous environments as a consequence of the hierarchical nature of their assembly: macroaggregates contain microaggregates, which either formed inside the macroaggregate or existed before and were integrated when the aggregate was formed, and pieces of particulate organic matter as well as interior pore spaces (Figure 1a). Selection pressures due to the different environmental conditions in the aggregate interiors and strong species interactions, especially competition (Barraclough, 2015), may lead to a decrease of genetic diversity in enclosed populations. In addition to natural selection, genetic drift will likely be a significant evolutionary force that further reduces genetic diversity (Figure 1b). Isolation of the enclosed microbial community prevents gene flow from countering the negative effects of natural selection and genetic drift on its genetic diversity. However, spatial heterogeneity within the aggregate and increased mutation rates due to elevated stress levels (for example, due to nutrient depletion and toxin accumulation) may, to a certain extent, maintain or increase genetic diversity within the aggregate. For example, in Escherichia coli genomes mutation rates can drastically increase and this generates variants including those with diverse metabolic capabilities (for example, Saint-Ruf et al., 2014). In addition, selection pressures may be highly temporally and spatially dynamic among aggregate interiors within a population of aggregates due to differences in the aggregation process and the particular founding subsample of the microbiome included in the aggregate.

Aggregate disintegration: natural selection and hybrid genotypes

Upon disintegration of soil aggregates (Figure 1b), the enclosed microbial communities are released and subjected to environmental conditions that differ from the aggregate interior. In addition, they will interact with the microbial community of the bulk soil including released communities of neighboring aggregates that disintegrated. Merging of these differentiated communities (genetic admixture) may increase the overall genetic diversity of the soil microbiome and, especially in the case of sexually reproducing species, has the potential to generate hybrid genotypes with higher levels of fitness (heterosis or hybrid vigor) that may spread through the soil.

Overall evolutionary consequences for soil biota diversity: aggregates as the cradle of soil biodiversity? It should be clear from the discussion above that the continuous formation and disintegration of vast amounts of soil aggregates catalyzes evolutionary processes in the soil microbial community and may shape its evolutionary pathway. Evolutionary processes within each single aggregate are thereby likely to a decrease the genetic diversity of the enclosed microbial community. However, we hypothesize that the effect of having soil aggregates-compared to a well-mixed habitat or even a statically spatially heterogeneous habitat-will lead to the generation of higher levels of genetic diversity in the soil biota as a whole. The main reason is the concurrent nature of a great diversity of starting conditions, founder effects and subsequent independent evolutionary trajectories pursued by the microbial populations inside of aggregates.

\section{How to study microbial evolution in soil aggregates}

Empirical as well as modeling approaches can be used to study the evolutionary aspects of soil aggregation. Computational modeling allows to study systems for which experimental solutions are not readily available in microbial ecology (Widder et al., 2016). However, basic features will likely have to be first explored by generating empirical data.

Whole-genome sequencing has opened paths to the study of evolution of pathogenic bacteria within the human body, as recently reviewed by Didelot et al. (2016) and Biek et al. (2015). These methods have recently become feasible because of the greatly reduced sample analysis costs. Such approaches and 
Table 2 Examples of questions arising from the study of soil aggregates as unique environmental compartmentalizations for microbial evolution
Theme and question
General approach
Do microbial phylotypes that are most strongly involved in the formation and/or stabilization of soil aggregates (for example, Lehmann and Rillig, 2015) also profit most in terms of evolutionary diversification, and eventually fitness, from their temporary inclusion in soil aggregates?
What are the dynamics of community composition following formation of an aggregate, and how does this interact with evolutionary change?
How do fungi behave in evolutionary terms, organisms which due to their hyphal growth form can integrate across aggregate and non-aggregate habitats?
Soil aggregates as cradles of microbial biodiversity in soil: basic conditions
Laboratory incubations informed by knowledge of microbial
phylotypes most important for soil aggregate formation
Time-course community profiling coupled with higher resolution tracking of evolutionary change in selected community members
Laboratory experiments with isolates of filamentous fungi
Significance in the context of applications Agriculture: does tillage, by disrupting soil aggregates, reduce an important source of evolutionary changes in soil microbes? Pollution ecology: will potentially smaller concentrations of pollutants in aggregate interiors (due to diffusion limitation) permit longer time periods for evolution of resistance?
Use of long-term agricultural experimental facilities
Laboratory incubations including monitoring of pollutant con- centrations within aggregates with a variety of methods (Nano- SIMS, X-ray CT, quantum dot labeling); testing of relative resistance of phylotypes from aggregate interiors

Significance for the understanding of consequences of factors of global change

Do agents of global change, such as warming or exposure to elevated atmospheric $\mathrm{CO}_{2}$, via changing the soil aggregation cycle, leave an imprint on microbial evolutionary trajectories? Will ecological changes that occur with a different time course (for example, gradual vs abrupt increase of a driver) be differentially buffered inside of aggregates compared to outside? Can global change factors that reduce soil aggregation, also reduce the microbial capacity for adaptation? Use of existing global change experimental facilities.

Laboratory experimentation with dedicated manipulation of time course of ecological drivers

Isolation of microbes from ongoing experiments coupled with laboratory experimentation

Abbreviation: CT, computed tomography.

the toolbox developed for studies in a more clinical/ epidemiological context (that is, isolation, genome sequencing, assembly and comparative genome analysis) could also be brought to bear on the situation in soil aggregates. Specifically, strains of a particular target species could be isolated from various co-occurring soil aggregates (similarly to Vetsigian et al. (2011) or Scheinin et al. (2015)) and then subjected to whole-genome sequencing. In addition, the recently developed maximum-depth sequencing approach and error-corrected highthroughput sequencing method could be employed (Jee et al., 2016). Often isolation of microbes will not be possible; in such cases, single-cell genomics or metagenomics techniques would be the method of choice, coupled with the ever-increasing arsenal of omics techniques for functional characterization (for example, proteomics, metabolomics). Ideally, such sequencing should be accompanied by assessments of microbial community composition in aggregates, and a monitoring of environmental microsite conditions to facilitate a better understanding of the mechanisms underlying such changes.

In addition to purely observational sequencingbased approaches, also direct experimental approaches could be pursued. Such experiments should start with using model bacterial systems with well-characterized players (for example, in terms of reference genomes) that occur in soils, such as Bacillus subtilis or Pseudomonas aeruginosa. Aggregates could be artificially formed in the lab, encapsulating starting cultures of such strains with known characteristics, and then evolution with and without aggregate association could be directly compared. Given the absence of other microbes, strains could be easily re-isolated for whole-genome sequencing. Such systems could then used as a starting point to increase complexity in a stepwise fashion, for example by incrementally including other members of the microbial community, including other bacteria and also fungi, and also representatives of other trophic levels. Eventually, questions of a more complex or applied nature, or in the context of global change could be tackled (Table 2).

\section{Conclusions}

There is a great general need to build a predictive understanding of the ecological and evolutionary dynamics of microbial communities (Widder et al., 2016). For soils, this is especially true, given the importance of soil microbes in global biogeochemical cycles, human and ecosystem health (Wall et al., 2015), for agricultural sustainability and the risks in store in the future in terms of extinctions of soil 
microbial taxa (Veresoglou et al., 2015). Clearly, in microbial systems, it is important to consider evolutionary processes when studying communities due to the frequent coincidence of ecological and evolutionary timescales. Explicitly focusing on soil aggregates holds great promise of uncovering a possible major element mediating evolutionary change within soil microbial communities.

\section{Conflict of Interest}

The authors declare no conflict of interest.

\section{Acknowledgements}

MCR acknowledges funding from Deutsche Forschungsgemeinschaft (DFG) and from the ERC Advanced Grant Gradual Change. We thank the reviewers for their helpful comments.

\section{References}

Barraclough TG. (2015). How do species interactions affect evolutionary dynamics across whole communities? Annu Rev Ecol Evol Syst 46: 25-48.

Biek R, Pybus OG, Lloyd-Smith JO, Didelot X. (2015). Measurably evolving pathogens in the genomic era. Trends Ecol Evol 30: 306-313.

Cordero OX, Datta MS. (2016). Microbial interactions and community assembly at microscales. Curr Opin Microbiol 31: 227-234.

Crawford JW, Harris JA, Ritz K, Young IM. (2005). Towards an evolutionary ecology of life in soil. Trends Ecol Evol 20: 81-87.

De Gryze S, Six J, Merckx R. (2006). Quantifying waterstable soil aggregate turnover and its implication for soil organic matter dynamics in a model study. Eur J Soil Sci 57: 693-707.

Dechesne A, Wang G, Gülez G, Or D, Smets BF. (2010). Hydration-controlled bacterial motility and dispersal on surfaces. Proc Natl Acad Sci USA 107: 14369-14372.

Didelot X, Walker AS, Peto TE, Crook DW, Wilson DJ. (2016). Within-host evolution of bacterial pathogens. Nat Rev Micro 14: 150-162.

Jee J, Rasouly A, Shamovsky I, Akivis Y, Steinman SR, Mishra B et al. (2016). Rates and mechanisms of bacterial mutagenesis from maximum-depth sequencing. Nature 534: 693-696.
Lehmann A, Rillig MC. (2015). Understanding mechanisms of soil biota involvement in soil aggregation: a way forward with saprobic fungi? Soil Biol Biochem 88: 298-302.

Rillig MC, Lehmann A, Aguilar-Trigueros CA, Antonovics J, Caruso T, Hempel S et al. (2016). Soil microbes and community coalescence. Pedobiologia 59: 37-40.

Saint-Ruf C, Garfa-Traore M, Collin V, Cordier C, Franceschi C, Matic I. (2014). Massive diversification in aging colonies of Escherichia coli. J Bacteriol 196: 3059-3073.

Scheinin M, Riebesell U, Rynearson TA, Lohbeck KT, Collins S. (2015). Experimental evolution gone wild. $J$ Soc Interface 12: 1-5.

Sexstone AJ, Revsbech NP, Parkin TB, Tiedje JM. (1985). Direct measurement of oxygen profiles and denitrification rates in soil aggregates. Soil Sci Am J 49: 645-651.

Six J, Bossuyt H, Degryze S, Denef K. (2004). A history of research on the link between (micro)aggregates, soil biota, and soil organic matter dynamics. Soil Tillage Res 79: 7-31.

Veresoglou SD, Halley JM, Rillig MC. (2015). Extinction risk of soil biota. Nat Commun 6: 1-10.

Vetsigian K, Jajoo R, Kishony R. (2011). Structure and evolution of Streptomyces interaction networks in soil and in silico. PLoS Biol 9: e1001184.

Vos M, Wolf AB, Jennings SJ, Kowalchuk GA. (2013). Micro-scale determinants of bacterial diversity in soil. FEMS Microbiol Rev 37: 936-954.

Wall DH, Nielsen UN, Six J. (2015). Soil biodiversity and human health. Nature 528: 69-76.

Whitman WB, Coleman DS, Wiebe WJ. (1998). Prokaryotes: the unseen majority. Proc Natl Acad Sci USA 95: 6578-6583.

Widder S, Allen RJ, Pfeiffer T, Curtis TP, Wiuf C, Sloan WT et al. (2016). Challenges in microbial ecology: building predictive understanding of community function and dynamics. ISME J 10: 2557-2568.

(c) (1) ()(2) This work is licensed under a Creative Commons Attribution-NonCommercialShareAlike 4.0 International License. The images or other third party material in this article are included in the article's Creative Commons license, unless indicated otherwise in the credit line; if the material is not included under the Creative Commons license, users will need to obtain permission from the license holder to reproduce the material. To view a copy of this license, visit http://creativecommons.org/ licenses/by-nc-sa/4.0/

(C) The Author(s) 2017 